\title{
Necropolítica de vivienda: 40 años desmantelando la “informalidad" en Madrid (1979-2019)
}

Eva Álvarez-de Andrés. Universidad Politécnica de Madrid, Madrid, España.

RESUMEN | En un contexto de creciente mercantilización y financiarización de la vivienda, la informalidad se ha convertido en la única alternativa, por precaria que pueda llegar a ser, para quienes han quedado excluidos del sistema formal. Desde la crisis económica de 2008, este fenómeno ha reaparecido en Europa, despertando un interés creciente. En este artículo se ha revisado el concepto de "informalidad" y se han analizado las políticas llevadas a cabo en la Comunidad de Madrid (España) en relación con los asentamientos autoproducidos en los últimos cuarenta años (19792019). Los resultados obtenidos muestran cómo todas las políticas han ido encaminadas a la erradicación de estas formas de autoproducción del espacio. No obstante, los planes implementados han variado en función del valor que las comunidades afectadas tenían para el poder establecido, forzándose la desaparición de aquellas que no entran en las formas de producción y consumo del sistema capitalista (necropolítica).

PALABRAS ClAVE | marginalidad, política habitacional, desigualdad social.

ABSTRACT | In a context in which access to housing has been increasingly commodified and financialized, "informality" becomes the only alternative-however precarious this may be-for those who have been excluded from the "formal" system. Since the beginning of the economic crisis in 2008, this phenomenon is reappearing in Europe, attracting more and more interest. In this article, we review the concept of "informality", and analyze the policies implemented in the Community of Madrid (Spain) in relation to the self-produced settlements in the last 40 years (1979-2019). The results show how all policies have aimed at the eradication of any form of self-production of space. However, the implemented plans have varied depending on the value that the affected communities had for the established power, forcing the disappearance of those that do not enter into the forms of production and consumption of the capitalist system (necro-policy).

KEYWORDS | marginality, housing policy, social inequality. 


\section{Introducción}

uN-Habitat (2007) estima que, para el año 2020, 1392 millones de personas vivirán en asentamientos "informales", el doble de lo que se estimaba en 1990 (tabla B3, p. 352). Desde la crisis de 2008, se ha incrementado la mercantilización (Harvey, 2012) y la financiarización de la tierra y la vivienda en todo el mundo (Rolnik, 2018). Estos bienes resultan cada vez más inaccesibles para los grupos de más bajos ingresos, debido a la brecha entre la subida de los precios de mercado y la precarización de las condiciones laborales (Piketty, 2015; Portes, Roberts, \& Grimson, 2005;). Tal situación ha contribuido al desarrollo de formas alternativas de acceso al espacio, la vivienda o servicios básicos como el agua o la energía, constituyéndose en un modo significativo de construcción de la ciudad (Davis, 2001; Vaccotti, 2017). Se trata de un modelo de autoproducción del espacio que ha llegado a ser, para muchas personas, el único modo de acceso a la vivienda o a los servicios básicos de la ciudad, aunque sea en condiciones muy precarias (Secchi, 2013).

Las diferentes formas de autoproducción del espacio, comúnmente denominadas "informales", han venido dándose en mayor o menor medida a lo largo de toda la historia, y ponen de relieve cómo, a pesar de los esfuerzos invertidos por formalizar la vida urbana y su desarrollo, el sistema formal parece representar apenas la punta del iceberg de un subsistema desregulado, parcialmente regulado o no regulado (Herrle \& Fokdal, 2011). Watson (2009) afirma que, tras la crisis financiera, la informalidad se ha convertido cada vez más en la norma y no en la excepción, un fenómeno que está reapareciendo en el Norte Global y despertando un interés creciente (Alfaro et al., 2018; Martínez \& García, 2015; Netto, Fitzpatrick, Sosenko $\&$ Smith, 2015).

El concepto de informalidad se ha venido definiendo y redefiniendo desde los años setenta. En un principio se consideraba únicamente como apropiado para definir el modo de supervivencia de los pobres urbanos, entendido como un sector ya sea de la economía o de la ciudad, que podía ser analizado desde fuera y de forma aislada sin tener en cuenta sus interrelaciones con otros sectores. En los ańos ochenta y noventa, el término "informal" fue adoptado por los expertos en vivienda y planificación, y aplicado a los asentamientos autoproducidos por las comunidades de bajos ingresos, considerándolos como espacios de la ciudad "desregulados", "no planificados" o "ilegales", destinados a aquellos que han quedado excluidos de las políticas de vivienda (Roy, 2010). A pesar de los trabajos de Turner (1967, 1968, 1972) en que muestra que estos asentamientos no son ni desorganizados ni irregulares, el término "asentamientos informales", o alguno de sus eufemismos, como "asentamientos espontáneos", se ha seguido aplicando. Estudios como los de Durand-Lasserve y Royston (2002) han puesto de relieve cómo la "ilegalidad" se ha venido utilizando a manera de pretexto para llevar a cabo procesos masivos de desalojo de los sectores más desposeídos. La "legalización” y la "regularización" defendidas por Hernando de Soto (2000) se convierten entonces en la estrategia que se debiera seguir para incluir estos espacios en el sistema urbano "formal". Estos posicionamientos, sin embargo, han tendido a obviar las relaciones entre los 
diferentes sectores y actores urbanos que constantemente definen y redefinen lo que se considera o no aceptable y lo que es legal o no lo es.

Desde esta perspectiva, cada vez más autores (Alfaro et al., 2018; Álvarez, Fernández, \& Smith, 2015; Herrle \& Fokdal 2011; Roy, 2010) cuestionan la utilidad del término "formalidad" y ponen el foco en la necesidad de analizar el fenómeno desde su complejidad; es decir, desde una mirada más holística que permita vincular los factores económicos, sociales y de gobernanza que lo conforman. Herrle y Fokdal (2011) sostienen que es necesario superar la dualidad implícita en el término "informalidad" y transitar hacia una definición del fenómeno en que se lo conceptualice como un proceso de negociación entre actores, caracterizado por el acceso de los mismos a los recursos, el poder y la legitimidad. En este sentido, Álvarez, Cabrera y Smith (2019) afirman que la informalidad es ante todo una cuestión política, que tiene que ver con el lugar que se ocupa dentro del espacio social y el modo de vinculación que se tiene con el resto de las estructuras de poder.

Según Alfaro et al. (2018), en lo que concierne a la informalidad, los patrones de relación en que ella se da han venido marcados por la desigualdad, con lo que esta situación implica de constreñimiento y reducción de la capacidad de negociar y de influir en los procesos de toma de decisiones. En tal sentido, Varley (2013) afirma que estos fenómenos son el reflejo de una relación rota entre el Estado y los ciudadanos, una ruptura en ocasiones institucionalizada por parte del Estado que, bajo el etiquetado selectivo de ilegalidad o irregularidad, niega y anula las comunidades de bajos ingresos y sus formas de producción del espacio.

Como se viene señalando históricamente, la ciudad sigue siendo el reflejo de una lucha entre actores con desiguales capacidades de negociación y de acceso a los recursos (Harvey, 2012; Jenkins, Smith, \& Wang, 2007; Lefebvre, 1969). Ello remite a una lucha continua entre prácticas institucionalizadas y prácticas de autoorganización (García-Calderón \& Janoschka, 2016), cuyo desenlace varía en función de los diferentes contextos políticos, económicos, sociales y culturales en que se dé (Herrle \& Fokdal, 2011; Jenkins et al., 2007).

No obstante, décadas de investigaciones demuestran que las iniciativas con capacidad de producir cambios estructurales han sido aquellas en las que las diferentes prácticas de autoproducción del espacio han logrado el reconocimiento y el apoyo de las instituciones locales y de la sociedad en su conjunto (Batley \& Mcloughlin, 2010; Devas et al., 2001; Nickson \& Franceys, 2003; Roy, 2011; Wild, Chambers, King, \& Harris, 2012). La coproducción se considera cada vez más como la forma más eficiente y sostenible de asegurar el acceso a bienes y servicios, en tanto que constituye el fruto de las contribuciones tanto de residentes como de agentes públicos o privados (Albrechts, 2013; Batley \& Mcloughlin, 2010; Mitlin, 2008; Mitlin \& Bartlett, 2018; Satterthwaite \& Mitlin, 2014; Verschuere, Brandsen, \& Pestoff, 2012). No obstante, Roy y AlSayyad (2004) advierten del peligro de que este enfoque sirva para que los Estados y las administraciones locales se desresponsabilicen y dejen por ello de cumplir su función en tanto garantes de los derechos del conjunto de la sociedad y, en particular, de los grupos más desfavorecidos.

Por su parte, Álvarez et al. (2015) ponen de manifiesto que la falta de reconocimiento persistente de estos procesos por parte de algunos Estados, y la falta 
de apertura hacia procesos de coproducción, no es una posición naïf, ya que les permite seguir ejerciendo presión sobre las poblaciones de más bajos ingresos mediante procesos cíclicos de abandono y gentrificación; como señala Shiva (2005), permite que otros se apropien indebidamente de su riqueza y sus recursos. Abufhele (2019) muestra cómo tampoco es casual la identificación de asentamientos autoproducidos en tanto que "territorios de pobreza", entendidos como espacios despolitizados habitados por sujetos pasivos a la espera de ser beneficiarios de la política gubernamental, cuyo fin último -según Procacci (1991) - no sería la eliminación de la pobreza, ni mucho menos de sus causas estructurales, sino la eliminación de formas de vida que no están alineadas con el proyecto social, político y económico dominante, o que lo subvierten.

Lopes de Souza (2012) señala que, pese a que estas formas de producción del espacio son residuales en el Norte Global, no dejan de ser espacios disidentes que interrumpen el proceso de acumulación del capital y ponen en cuestión uno de los pilares fundamentales del sistema de acumulación capitalista: la propiedad privada. En este sentido, García-Calderón y Janoschka (2016) sostienen que estos espacios autogestionados se convierten en un desafío a los intereses del capital y una transgresión a las normas impuestas por el Estado. Por ello, a pesar de que estas formas de acceso temporalmente autónomo (Bey, 1991) son el único modo de acceso a la vivienda para quienes han quedado excluidos del acceso a través del mercado o del Estado, siguen siendo estigmatizadas y perseguidas hasta forzar su desaparición.

Y es en relación a esa política de la desaparición donde el término "necropolítica”, acuñado por el filósofo Mbembe (2003), cobra interés en este trabajo. Para Mbembe (2003), la necropolítica es la política cuyo punto de partida es la concepción de que la vida de un individuo o una comunidad tiene valor en la medida en que lo tiene para el poder establecido. A los no rentables, que no entran en las formas de producción y consumo del sistema capitalista, se les podría dejar morir, o dejar desaparecer. Mbembe entiende la necropolítica como un proceso biopolítico (Foucault, 2003); es decir, como un modo de ejercer poder sobre un determinado cuerpo social mediante prácticas que contribuyen a invisibilizar, precarizar y erradicar las formas de vida de los excluidos del sistema. Para Valverde Gefaell (2015), se trata de una política para forzar la desaparición, para imposibilitar las formas de vida de las comunidades de excluidos que, sin querer y sin saberlo siquiera en la mayoría de los casos, solo existiendo ponen en evidencia la crueldad del neoliberalismo y sus desigualdades.

El objetivo de este artículo es analizar las políticas llevadas a cabo en la Comunidad de Madrid (CM), en España, en relación con los asentamientos autoproducidos durante los últimos cuarenta ańos (1979-2019). Los resultados obtenidos muestran cómo, en los casos objeto de estudio analizados, las políticas y acciones han ido encaminadas a forzar la desaparición de estas formas de autoproducción del espacio (Procacci, 1991; Valverde Gefaell, 2015). La respuesta implementada ha variado según el valor que las comunidades afectadas tenían para el poder establecido, forzándose la desaparición de aquellas que no entran en las formas de producción y consumo del sistema capitalista (necropolítica). 
El artículo se organiza como sigue. Tras esta primera sección de introducción, se presenta el método de investigación utilizado para el análisis de las políticas implementadas por la см en relación con los asentamientos autoproducidos entre 1979 y 2019; a lo largo de los dos siguientes apartados se presentan los resultados obtenidos, para finalmente extraer conclusiones a partir de la discusión cruzada de dichos resultados.

\section{Método, selección de los casos de estudio, y recogida y análisis de la información}

Como se ha señalado en la introducción, el objetivo de este artículo es analizar las políticas llevadas a cabo en la CM, en España, en los últimos cuarenta años (19792019) en relación con los asentamientos autoproducidos. Este trabajo se ha basado en el método de casos de estudio (Yin, 1994) y se ha partido de la teoría de la estructuración de Giddens (1984). Desde este enfoque, la investigación se ha centrado en el análisis de los patrones de relación entre actores y del modo en el que dichos patrones han determinado el acceso a la vivienda y los servicios básicos de la ciudad (Alfaro et al., 2018; Herrle \& Fokdal, 2011).

Para ello se han analizado, en primer lugar, las políticas llevadas a cabo en Madrid frente a los asentamientos autoproducidos desde 1979 hasta 2015. Se distingue entre dos procesos que se dieron de forma paralela en dicho periodo: por una parte, el Plan de Remodelación de Barrios (РRв) (1979-1998), en tanto respuesta a la demanda de vivienda digna por parte de la clase obrera al inicio del periodo democrático; y por otra, las políticas destinadas a atender a la población considerada como "marginal", que se planificaron de forma paralela al PRB y se fueron desarrollando hasta el cierre del Instituto de Realojamiento de Integración Social (IRIS) en 2015. En segundo lugar, se ha analizado el proceso actual de desmantelamiento de uno de los últimos asentamientos autoproducidos de la CM, el de Las Sabinas (Móstoles) (2013-2019).

Se considera que la comparación de los resultados obtenidos respecto a los tres procesos seleccionados puede contribuir a responder a las siguientes cuestiones:

- ¿Hasta qué punto la política de intervención en Madrid, en relación con los asentamientos autoproducidos ("informales"), ha ido encaminada a la erradicación de estas formas alternativas de producción del espacio?

- ¿En qué medida las respuestas implementadas han venido determinadas por el valor que tienen las comunidades afectadas para el poder establecido? Y la falta de valor o de reconocimiento, ¿ha supuesto la implementación de políticas de desaparición (necropolíticas)?

La investigación se ha llevado a cabo en cuatro fases: inmersión (descripción), recogida de información sistemática (clasificación), procesado de la información (conexión) y extracción de conclusiones (producción de conocimiento) (Frediani, 2007). El proceso de inmersión se inició a finales de 2013 con la puesta en marcha del proceso de investigación-acción en Las Sabinas (Móstoles). Desde 2014 se ha venido recogiendo de forma sistemática la palabra y la experiencia de las familias afectadas por este proceso (cien familias), con lo que ello ha supuesto conocimiento 
de la palabra y la experiencia de quienes se ven afectados por estas políticas. Además, se ha participado en diversas reuniones y se han realizado entrevistas semiestructuradas a los responsables políticos (alcalde, concejales de vivienda y servicios sociales) y a los técnicos involucrados (servicios sociales de Móstoles, de la Agencia de la Vivienda Social, Avs). En un segundo momento, se ha trabajado en analizar la evolución de las políticas implementadas a lo largo de los últimos cuarenta ańos con respecto a los asentamientos autoproducidos en la $\mathrm{CM}$. Con tal fin se ha recogido la información disponible sobre los procesos precedentes (1979-2015), y se han realizado entrevistas semiestructuradas con los actores que participaron activamente en dichos procesos, en tanto líderes locales y expertos vinculados al PRB (asociaciones de vecinos, profesionales, etc.), así como con técnicos que trabajaron en las instituciones involucradas como el IRIs (clasificación). Posteriormente se ha analizado la información recogida atendiendo tanto al tipo de vínculos generados, como a las formas de acceso a los recursos (vivienda, servicios básicos, etcétera) derivadas de dichos vínculos (conexión). Los resultados obtenidos se han recogido en planos, tablas y flujogramas.

FIGURA I | Flujograma para la representación de las políticas implementadas FUENTE: ELABORACIÓN PROPIA



El flujograma (figura 1) se ha utilizado para representar la variación de las políticas implementadas en cada caso en relación con el reconocimiento, entendido como el valor que tiene o deja de tener una comunidad para el poder establecido, con el fin de visibilizar la existencia de necropolíticas. Además, permite mostrar variaciones respecto de otros parámetros, como la representación o la centralidad. Se entiende la representación como la capacidad de una determinada comunidad para participar en el proceso de toma de decisiones e incidir en el mismo; y la centralidad, como la posición física que ocupa un determinado asentamiento con respecto al centro, así como la importancia que la transformación del asentamiento tiene para la agenda política. 
Finalmente se ha procedido a la comparación de los casos y la extracción de conclusiones (producción de conocimiento).

A continuación se exponen los resultados del análisis en relación con los casos de estudio seleccionados. Se presentan, en primer lugar, los resultados de los procesos implementados entre 1979 y 2015, desde el PRB a las políticas implementadas para atender a la población "marginal", para pasar seguidamente a la presentación de los resultados del proceso de desmantelamiento de Las Sabinas (Móstoles) (20132019). Finalmente se presenta la discusión cruzada de los resultados y la extracción de conclusiones.

\section{Del Plan de Remodelación de Barrios (PRB) a las políticas de "realojamiento" de la "población marginal” (1979-2015)}

\section{Antecedentes (1936-1979)}

A finales de los años cincuenta, fruto de los procesos migratorios campo-ciudad, los asentamientos autoproducidos llegaron a suponer el 16,6\% del suelo residencial en Madrid (Carmona \& Rodríguez, 2007; Lago, 2014). Durante el periodo franquista, la administración persiguió la autoconstrucción y desatendió la demanda de estas familias dirigida a lograr acceso a una vivienda digna y a los servicios básicos, alegando que se trataba de barrios construidos de forma "ilegal" en suelo rústico.

En este contexto de abandono institucional, en torno a 300 mil personas siguieron autoconstruyendo sus viviendas y autogestionando su acceso a los servicios básicos. Con el tiempo, estos barrios inicialmente periféricos fueron ganando centralidad y se convirtieron en lugares de atracción para los nuevos desarrollos. En 1963, el Plan General de Ordenación Urbana (PGOU) cambió la calificación de estos suelos, de rústicos a urbanizables. La expectativa de nuevas plusvalías dio lugar a un sin fin de propuestas de Planes Parciales que, bajo el pretexto de mejorar las condiciones de higiene y el entorno urbano, suponían de facto la expulsión de las familias que los habitaban hacia nuevas periferias, a cambio de una ínfima indemnización.

Los vecinos de estos barrios, que habían ido ganando capacidad de autoorganización a través de los procesos de mejora colectiva de sus barrios, no estaban dispuestos a perder los bienes y vínculos que habían ido generando a lo largo de décadas. Las asociaciones de vecinos y quienes les apoyaban (colectivos de estudiantes, organizaciones cristianas de base, organizaciones políticas emergentes que se oponían al régimen franquista, etcétera), se movilizaron rechazando los planes oficiales y reclamando “ivivienda digna, aqui y ahora?'. Sus luchas fueron ganando legitimidad y lograron instaurar la idea de que existía una "deuda social" con estas familias, que habían sido la mano de obra que había construido la ciudad de Madrid, y que a cambio se les debía permitir permanecer en la periferia que habían ido colonizando y que reclamaban como suya.

El Plan de Remodelación de Barrios (PRB) (1979-1998)

En 1979, en el contexto de una democracia recién estrenada, se logró poner en marcha el Plan de Remodelación de Barrios (PRB), una propuesta liderada por las asociaciones de vecinos que contó desde su inicio con el apoyo del nuevo 
Ayuntamiento democrático y del Ministerio de Vivienda. Además, para la industria de la construcción, sumergida en la crisis económica de los años setenta, el plan suponía una inyección de fondos públicos y, por tanto, una importante oportunidad de reactivación del sector.

El PRB fue un proceso de más de quince años de negociaciones entre el movimiento vecinal organizado, los técnicos encargados del diseño de las viviendas y de los nuevos barrios, y los diferentes organismos de la administración que intervinieron a lo largo del proceso (Rodríguez-Villasante et al., 2009). Los conflictos fueron inevitables, pero gracias a los mecanismos de participación establecidos entre los distintos actores se pudo llegar a consensos y a fijar mecanismos de seguimiento adecuados.

La Administración fue la encargada de proveer los fondos para la expropiación del suelo, la construcción y financiación de las nuevas viviendas, los equipamientos e infraestructuras, etcétera. Las organizaciones vecinales elaboraron censos a partir de los cuales se fueron analizando y definiendo respuestas diferenciadas para los distintos casos. El derribo y realojo en las nuevas viviendas se organizó de forma que todo el mundo pudiera permanecer en su barrio.

Al concluir el PRB, se había realojado in situ a 150 mil personas procedentes de treinta barrios de características muy diferentes. Se habían construido 39 mil viviendas con una inversión por parte de la Administración del orden de unos 50 mil euros por familia realojada. Más allá de estos datos, se consideran entre los principales logros de este proceso (Castro \& Molina, 1996), los siguientes:

- El reconocimiento de la "deuda social” y la participación vecinal activa a lo largo de todo el proceso.

- La apertura por parte del gobierno nacional, regional y local y su aceptación de los mecanismos de control vecinal implementados.

- La coordinación entre el movimiento vecinal organizado, los técnicos y la administración.

- El realojo en el propio barrio y, por tanto, la posibilidad para los vecinos de consolidar sus bienes y vínculos.

- El acceso a una vivienda de calidad de en torno a $100 \mathrm{~m}^{2}$ en condiciones de seguridad y permanencia (en régimen de propiedad).

- La compensación económica por el suelo y la vivienda autoconstruida como parte del pago para la nueva vivienda, disminuyendo el coste de la nueva hasta un $15 \%$ del precio final.

- Las condiciones de financiación accesibles para las familias con bajo nivel de renta (hipotecas a treinta ańos con cuotas por debajo del 10\% de los ingresos familiares y anticipos sin intereses por el $75 \%$ del coste de la vivienda).

- Soluciones adaptadas a cada barrio y a cada caso dentro de un mismo proceso colectivo.

- La construcción de barrios enteros de forma participativa, con un alto grado de equipamiento y dotaciones, e integrados con el resto de la ciudad. 
En 1986, el porcentaje de familias residentes en asentamientos autoconstruidos había descendió al 9\%; de estas, en un 93\% eran familias gitanas que no eran propietarias de suelo, no pagaban alquiler, no disponían de rentas, etcétera. Se trataba de familias física, social y económicamente discriminadas, todo lo cual, en lugar de convertirse en un reto sociopolítico de integración, las dejó fuera del PRB (Montes, 1994).

Desde ese momento, el chabolismo ${ }^{1}$ en Madrid dejó de ser un problema generalizado que afectaba a amplios sectores de la sociedad y pasó a ser un problema de sectores “marginales”. En 1984, el Gobierno Regional fundó el Instituto de Vivienda de Madrid (IVIMA), orientado a promover vivienda en régimen de propiedad para las clases medias, al tiempo que se elaboró un plan para "controlar el fenómeno del chabolismo".

\section{Los planes de vivienda para la población "marginal” (1986-2015)}

En 1986 se creó el Consorcio para el Realojamiento de Población Marginal (CRPM) con el objetivo de "erradicar el chabolismo" de la ciudad de Madrid (situado en un $87 \%$ en los distritos del sureste), aprovechar el suelo liberado para los nuevos desarrollos de la ciudad, y resolver además los conflictos sociales generados entre las comunidades gitanas y los nuevos barrios. Se estimó que el proceso de erradicación del chabolismo se llevaría a cabo en un periodo máximo de cinco años, y se propusieron tres modalidades de realojo diferenciadas en función del grado de integración de la comunidad gitana afectada: vivienda en altura, Barrios de Tipología Especial (вте) о Campamentos Provisionales (СР).

No obstante, la falta de apoyo político y de disponibilidad del correspondiente parque habitacional llevó a que solo el $35 \%$ de las familias afectadas fuera realojado (Nogués, 2010); y de dichas familias, el $55 \%$ en вTE o en СР у no en vivienda en altura en sus propios barrios, como lo había solicitado el $82 \%$ de los afectados.

Estos barrios, si bien habían sido concebidos como provisionales, en algunos casos perduraron hasta seis décadas (El Mundo, 2015/03/07). Con el tiempo se convirtieron en guetos y fueron objeto de nuevos desmantelamientos llevados a cabo por las mismas administraciones que los habían promovido. Esto se debió fundamentalmente a que en ningún momento se consideró la necesidad de integrarlos física y socialmente. En la mayoría de los casos, se ubicaron en lugares inadecuados o insalubres y desconectados del resto de la ciudad y, por tanto, del acceso a equipamientos y servicios básicos. Según afirma Nogues (2010), "alguna familia prefería perder su derecho a vivienda con tal de no tener que seguir viviendo allí” (p. 284). En estos años, lejos de lograrse la erradicación del chabolismo, este se incrementó, estimándose en 1994 un aumento de más de mil nuevas viviendas autoconstruidas.

El fracaso de esta experiencia, así como los conflictos entre los distintos niveles de la administración para su gestión (estatal, regional y local), llevó a que se cerrara el CRPM y se creara el Instituto de Realojamiento e Integración Social (IRIS). El

Entendido como barrios autoproducidos, física, social y económicamente excluidos del sistema (villas miseria, favelas, etcétera). 
IRIS se constituyó en 1998, partiendo del reconocimiento de que todas las familias tenían derecho a una vivienda digna, entendida como un elemento clave del proceso de integración social, y por ello la primera acción que se llevó a cabo fue la realización de un censo. Además, se concibió como un instrumento de mediación entre la administración regional (CM) y local (Ayuntamientos), si bien los planes se siguieron llevando a cabo en exclusiva por la CM hasta 2006, fecha en la que inició la firma de convenios entre administración regional y ayuntamientos.

Por lo general, en estos convenios-programas se recogían tanto las obligaciones de las distintas administraciones involucradas (aportaciones económicas, acciones por desarrollar, etcétera), como los requisitos fijados para el realojo de las familias afectadas. Entre los requisitos se establecía un plazo mínimo de empadronamiento (en torno a cinco ańos desde la firma del convenio), así como no haber sido adjudicatarias de vivienda pública en los últimos veinte años. En dichos convenios no se fijaba una renta mínima para el acceso a la vivienda, si bien a la hora de la adjudicación de la misma sí se solicitaba dicha renta a las familias para asegurarse de que podían hacer frente a los pagos que implicaba el realojo en un piso (alquiler, comunidad y servicios de agua, luz, etcétera). Según Nogués (2010), entre 1998 y 2006 se desmantelaron 43 núcleos chabolistas y se adjudicaron 1223 viviendas, situadas en un $90,5 \%$ en el sur y sureste de la CAM (en los municipios de rentas más bajas). Se estima que la inversión en pisos por familia realojada fue de unos 110 mil euros, lo que equivalía a un piso en régimen de propiedad, si bien las familias fueron realojadas en régimen de alquiler. Según Nogués (2010), entre 2005 y 2006 se demolieron 421 chabolas y se realojó a 232 familias, lo que indica que, al menos en esos años, el $55 \%$ de las familias afectadas por procesos de realojo se vieron en la calle sin alternativa habitacional, y forzadas a "empezar de cero" en barrios chabolistas como La Cañada Real o en Las Sabinas (Móstoles), que nunca se incluyeron en los censos del IRIs.

Mientras las familias perdían su red social de apoyo mutuo y las ventajas de la centralidad que habían logrado tras décadas de autoproducción y resistencia, los nuevos suelos liberados, así como las viviendas concedidas para el realojo, pasaron a formar parte del patrimonio público. No existe información pública disponible acerca de cuántas de estas familias permanecen en la actualidad realojadas en sus viviendas en régimen de alquiler social. Esta cuestión es particularmente relevante tras producirse en 2013 la venta de 1860 viviendas sociales a "fondos buitre", ${ }^{2}$ a un $25 \%$ por debajo de su valor de mercado, por parte de la cam. Según el dictamen del tribunal de cuentas, ello "dio lugar a un menoscabo injustificado en el patrimonio público" (El Diario, 29/12/2018).

De acuerdo con fuentes no oficiales (El Plural, 20/07/2013), la última vivienda entregada a una familia chabolista por parte del IRIS se realizó en 2011. No obstante,

2 Se trata de una práctica de especulativa consistente en la compra, por parte de fondos de inversión extranjera, de vivienda pública a precios muy inferiores a su precio de mercado. En general, son fondos de capital riesgo que compran deuda de economías en problemas, cercanas a la quiebra, para posteriormente presionar y cobrar la totalidad del valor de esa deuda, además de los intereses por los ańos adeudados. (Andbank, Observatorio de Inversor, Espańa. https://www.andbank.es/ observatoriodelinversor/que-son-los-fondos-buitre-capital-riesgo/). 
entre 2011 y 2013, el IRIs adjudicó 278 viviendas a solicitantes de vivienda pública de la Dirección General de Vivienda y Rehabilitación, para un propósito que nada tenía que ver con los planes de realojo del IRIs.

En las casi tres décadas, desde la creación del CRPM al cierre del IRIS (1986-2015), se observa que, a diferencia del PRB:

- Estos procesos fueron liderados por las administraciones públicas. En el periodo del CRPM se llevaron a cabo sin participación alguna por parte de las familias afectadas. En el periodo del IRIs, la participación se limitó a la elección, por parte de las familias que cumplían los requisitos de los respectivos programasconvenio, de una vivienda de entre tres preseleccionadas por el IRIS.

- Apenas se realojó a 4 mil familias, es decir, un 10\% de las que se habían realojado en el PRB en la mitad de tiempo.

- En lo que respecta al CRPM, el 44\% de los realojos se llevó a cabo en BTE o CP, que con el tiempo se convirtieron en guetos y tuvieron que ser desmantelados por las mismas administraciones que los habían promovido.

- En lo que respecta al IRIs, de las 2 mil viviendas otorgadas, en un 90\% estaban situadas en el sureste de la CAM, en los municipios de más bajo ingresos, con lo que ello implica de refuerzo de la segregación socioespacial entre los municipios del norte y del sur de la cam.

- Las viviendas se adjudicaron en régimen de alquiler social, lo que suponía para las familias entre el $50 \%$ y el $100 \%$ de sus ingresos. No se dispone de información pública acerca de cuántas de estas familias han logrado seguir haciendo frente a los pagos y han podido seguir ocupando los pisos que les fueron otorgados. Asimismo, se desconoce en qué medida se vieron afectados por la crisis económica de 2008 y la venta de 1860 viviendas públicas a fondos buitre por parte de la CAM en 2013.

En 2015, se estimaba que alrededor de 4 mil familias seguían viviendo en asentamientos autoproducidos en la CAM, concentradas fundamentalmente en dos núcleos: La Cañada Real (el asentamiento autoconstruido más grande de toda Europa y que nunca se contempló en los programas del IRIS) y Las Sabinas, un asentamiento en el que en torno al $90 \%$ de las familias gitanas provenían precisamente de los programas de desmantelamiento de otros núcleos, como Las Mimbreras o El Salobral.

En este contexto, la См decidió poner en marcha la Agencia de Vivienda Social (AVs), integrando en el mismo organismo el IVIMA y el IRIs, dos instituciones que habían sido creadas para responder a la demanda de vivienda, demanda que no había hecho sino aumentar desde la crisis de 2008.

\section{Análisis de los resultados del periodo 1979-2015}

Los resultados obtenidos en el periodo 1979-2015 muestran cómo la política de intervención en Madrid, en relación con los asentamientos autoproducidos (informales), se encaminó a la erradicación de esta forma de producción del espacio (figura 2), que se redujo hasta un $75 \%$ (tabla 1 ). 



FIgURA 2 | Chabolismo de los años cincuenta y PRB

FUENTE: NOGUÉS, 2010

No obstante, se observa cómo los planes implementados variaron atendiendo al reconocimiento de las comunidades afectadas por parte del poder establecido. El PRB surgió como respuesta a las demandas de la clase obrera, que contaba con el reconocimiento de un gobierno democrático recién estrenado y cuya incorporación al sistema de producción y consumo capitalista se consideraba esencial. El PRB fue un plan de "formalización" del acceso a la vivienda, liderado por un movimiento vecinal organizado. Por ello, a pesar de la centralidad que habían ido ganando los terrenos ocupados, se logró el realojo in situ del 97\% de los afectados (figura 3), en viviendas en régimen de propiedad, y en condiciones adaptadas para cada caso y asequibles ( $<10 \%$ de la renta familiar). Sin embargo, el 3\% restante, principalmente población gitana fuertemente estigmatizada, quedó excluida de dicho proceso.
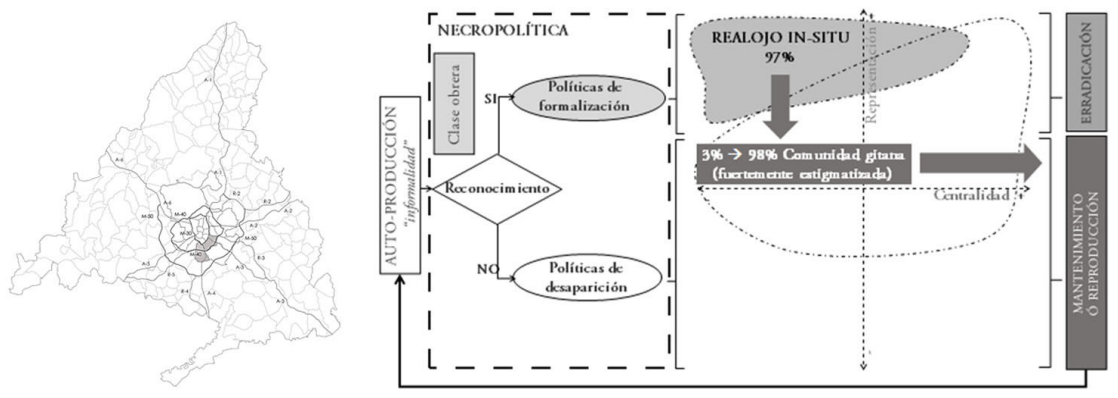

FIgURA 3 | Plano de situación del PRB y flujograma de las políticas implementadas

FUENTE: ELABORACIÓN PROPIA

Desde 1986, la autoproducción de vivienda (“informalidad”) en Madrid pasó a ser una cuestión de sectores "marginales". El CRPM se creó con el objetivo explícito de erradicar el chabolismo de la ciudad de Madrid. Se observa cómo, en las casi tres décadas desde la creación del CRPM al cierre del IRIS (1986-2015), las comunidades afectadas no contaron con el reconocimiento del poder establecido. Los planes se 
llevaron a cabo sin la participación de los afectados (representación). Se desconoce si esto se debió a la falta de organización de estas comunidades o a que sus demandas fueron ignoradas. No obstante, la consecuencia fue la implementación de políticas para forzar la desaparición de estas comunidades mediante planes de realojo gentrificados e inseguros (figura 4).
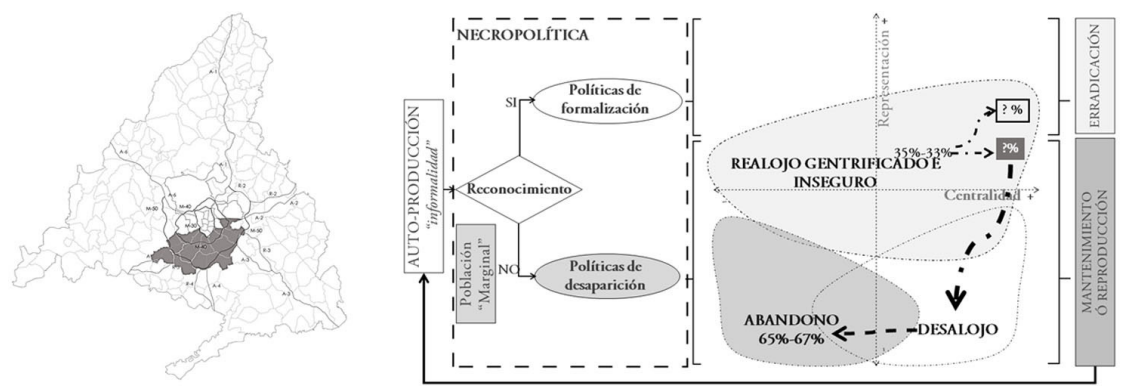

FIGURA 4 | Plano de situación de las viviendas de realojo otorgadas por el IRIS y flujograma de las políticas implementadas

FUENTE: ELABORACIÓN PROPIA

Se estima que en torno al $65 \%$ de las familias que vivían en asentamientos autoproducidos no fueron incluidas en estos procesos y, por tanto, se mantuvieron en la informalidad. No obstante, no se dispone de información oficial para saber:

- cuántas de las familias afectadas por estos procesos de realojo fueron desalojadas sin alternativa habitacional;

- y cuántas de las que fueron realojadas en vivienda en régimen de alquiler (con gastos del orden del 50\% al 100\% de los ingresos familiares) fueron con los ańos desalojadas por las mismas instituciones que las habían realojado (por impago o venta de vivienda pública); y, por tanto, cuántas volvieron a vivir en barrios autoproducidos como el de Las Sabinas en Móstoles, cuyo proceso de desmantelamiento pasamos a presentar a continuación.

\section{El desmantelamiento de Las Sabinas (Móstoles) (2015-2019)}

\section{Antecedentes (1980-2015)}

En 2013 se estimaba que el asentamiento de Las Sabinas estaba conformado por unas 375 familias repartidas en cuatro núcleos, entre los municipios de Móstoles (zonas 1 y 2) y Arroyomolinos (zonas 3 y 4), a unos 25 kilómetros al sur de Madrid. El convenio firmado en 2013 entre la CAM y el ayuntamiento de Móstoles comprendía la zona 1, habitada fundamentalmente por población inmigrante; y la zona 2 , habitada por población gitana proveniente en un $90 \%$ de anteriores procesos de desmantelamiento de barrios como Las Mimbreras o El Salobral, de los que las familias fueron desalojadas sin alternativa habitacional. 
En los dieciséis años de desarrollo de este núcleo, el abandono institucional ha sido la única respuesta por parte de las administraciones que deberían haber sido garantes de derechos. Hasta la fecha, se sigue sin disponer de fuentes de agua potable, servicio de transporte público, escuela, centro de salud, etcétera. La falta de algunos servicios públicos, como el de recogida de basuras, se convierte además en un elemento clave para la estigmatización de esta comunidad. En este contexto, las familias se preguntan "si no son parte de la sociedad como los demás, con los mismos derechos". A las dificultades cotidianas de vivir en estas condiciones, se suman los riesgos de incendios, las inundaciones, los cortes de luz por parte de la compañía eléctrica, etcétera.

Tras décadas de abandono, por fin se firmó el convenio de realojo en 2013 y se establecieron las obligaciones de las dos administraciones involucradas (aportaciones económicas, acciones por desarrollar, etcétera), así como los requisitos que deben cumplir las familias para ser realojadas, entre los que se fija un empadronamiento mínimo de cinco años en el municipio (y no en la CAM) y no haber sido adjudicatarios de vivienda pública en los últimos veinte años.

En el momento de la firma del convenio, los servicios sociales de Móstoles estimaban que el realojo afectaría a 272 familias, si bien solo 124 cumplían los requisitos establecidos. Por ello, desde la firma del convenio supuestamente de realojo, las administraciones partieron del desalojo, sin alternativa habitacional, del 45\% de las familias afectadas, algo que va en contra del artículo 47 de la Constitución Espańola, así como del artículo 11.1 del Pacto Internacional de Derechos Económicos, Sociales y Culturales (Pidesc) ratificado por el Estado español.

A pesar de que el convenio se firmó en 2013, en 2015 el Ayuntamiento de Móstoles seguía sin cumplir con su obligación de aportar los fondos; y sin que, por otra parte, la CAM exigiera dicho cumplimiento, por lo que la Avs heredó del IRIs el correspondiente programa-convenio, sin que se haya siquiera iniciado el proceso.

El plan de desmantelamiento de Las Sabinas (Móstoles) (2015-2019)

En 2015 se produjo un cambio de gobierno en el Ayuntamiento de Móstoles, lo que para las familias y quienes les apoyan supuso una oportunidad para reivindicar que se firmara un nuevo convenio, que contemplara el realojo de todas las familias, y que se aportaran los fondos correspondientes para que este pudiera ser ejecutado. Sin embargo, las administraciones acordaron que se ejecutara el convenio tal cual se firmó en 2013, sin que se permitiera a las familias participar en el proceso de negociación. Pese a que el realojo afectaba a los dos núcleos de Móstoles, se decidió que se ejecutara en su totalidad en el núcleo habitado por población gitana (zona 2 ), en el que se estimaba vivían unas cien familias de las cuales en torno al 50\% no cumplía los requisitos, sin que se previera ninguna alternativa para las mismas.

Durante cinco años, desde que se firmó el convenio en 2013 y se inició realmente el proceso en 2018, las familias han vivido en la más absoluta incertidumbre; no solo no se fijó una fecha para el inicio del realojo, sino que tampoco fueron informadas oficialmente sobre quiénes cumplían o no los requisitos, o dónde serían realojadas. Además, en esos años, el barrio siguió sin tener acceso a los servicios básicos y se imposibilitó a las familias hacer mejoras de las viviendas (por problemas 
estructurales, humedades, etcétera), al considerarse que estaban inmersas en un proceso de realojo.

Finalmente, a principios de 2018 se empezaron a adjudicar las viviendas, de las cuales se estima que se han entregado hasta el momento unas cuarenta; de estas, el $84 \%$ está al sureste de Madrid y un $68 \%$ en municipios situados de media a unos $50 \mathrm{~km}$ del centro. La ruptura con su tejido social y la distancia ha llevado a que un $20 \%$ de los realojados haya renunciado al piso o esté en proceso de hacerlo, con lo que ello supone de penalización a optar a una vivienda pública en los próximos diez años (según el último reglamento aprobado por la Avs). A todo ello se suma la dificultad de hacer frente al pago del piso, ya que la vivienda se adjudica en régimen de alquiler social, lo que, sumado a los gastos de comunidad, agua, luz, etcétera, supone entre el $50 \%$ y el $100 \%$ de los ingresos de las familias (la mayoría receptoras de Renta Mínima [RMI] o de pensiones no contributivas). A continuación, se recoge parte del testimonio de uno de los afectados:

Después de estar viviendo aquí trece años, sin agua, sin luz, con miedo a los incendios, viviendo entre las ratas (...) por fin nos llaman y nos dicen que nos han adjudicado la vivienda en Fuentidueña del Tajo. Es un pueblito a 97 kilómetros de Móstoles en el que no hay ni médico, ni instituto (...) lo que quieren es sacarnos de la sociedad (...). Y además el piso hay que pagarlo y, ¿cómo voy a pagarlo en un pueblo fantasma en el que no me puedo ganar la vida? Estoy desesperado, porque o cojo el piso o pierdo el derecho a una vivienda social durante los próximos diez años... iii Esta gente te mata de verdad!!! No imaginas la frustración y la impotencia que siento. iii Hemos pasado de vivir la ilusión del realojo a vivir un infierno!!! (Adjudicatario de vivienda de Las Sabinas, Móstoles).

En el caso de Las Sabinas (Móstoles), los resultados obtenidos muestran como:

- Las familias han quedado completamente excluidas del proceso de toma de decisiones.

- Tras cinco años de la firma del convenio, apenas un $11 \%$ de los afectados ha sido realojado.

- La AVs, gracias a la aportación de fondos europeos de desarrollo, ha comprado los pisos por un valor medio estimado de 114 mil euros por vivienda, si bien el valor de mercado de las viviendas adjudicadas se estima entre un $20 \%$ y un $50 \%$ por debajo del coste presupuestado.

- No obstante, las viviendas han sido otorgadas en régimen de alquiler social, lo que supone una aportación de entre el $50 \%$ y el $100 \%$ de los ingresos de las familias.

- El 84\% de las viviendas se encuentra, una vez más, en los municipios del sureste de la CAM, en periferias cada vez más alejadas del centro de Madrid, a unos 50 $\mathrm{km}$ de media.

- Se observa cómo las dificultades para hacer frente a los pagos, la ruptura de la red social de apoyo mutuo, el realojo en barrios de baja densidad que no permiten el desarrollo de actividades productivas, etcétera, han llevado a que, no 
habiendo pasado un año del realojo, un $20 \%$ de los afectados haya renunciado al piso o esté en proceso de hacerlo. Sin embargo, a pesar de que desde las instituciones no se ha tenido en cuenta la realidad de las familias para la elaboración y la ejecución del proceso de realojo, se culpabiliza a las víctimas penalizándolas con diez ańos sin acceso a una vivienda pública en caso de renunciar al piso.

En la actualidad sigue sin existir un censo preciso de cuántas familias viven en asentamientos autoproducidos en la CAM o en viviendas ocupadas. Dada la falta de respuesta efectiva por parte de las administraciones y el contexto de emergencia habitacional que se vive en España (Gutiérrez \& Vives-Miró, 2018), se prevé que la informalidad siga aumentando y acogiendo, entre otras, a las poblaciones no realojadas provenientes del proceso de desmantelamiento de Las Sabinas o del Sector 6 de La Cańada Real, en el que se está llevando a cabo un proceso similar.

\section{Análisis de los resultados del desmantelamiento de Las Sabinas (Móstoles)} (2015-2019)

En el caso de Las Sabinas, tras décadas de abandono institucional, en 2013 se firmó un convenio de realojo de una comunidad fuertemente estigmatizada y no reconocida por parte del poder establecido. El plan implicaba desde el inicio forzar la desaparición de dicha comunidad, al partir del desalojo sin alternativa habitacional del $45 \%$ de los afectados. El convenio tardó más de cinco años en empezar a ejecutarse, y se implementó únicamente en el núcleo que venía movilizándose desde 2014 (8\%). A pesar de las demandas de los afectados, las instituciones les han negado en todo momento su participación en el proceso de toma de decisiones (representación).
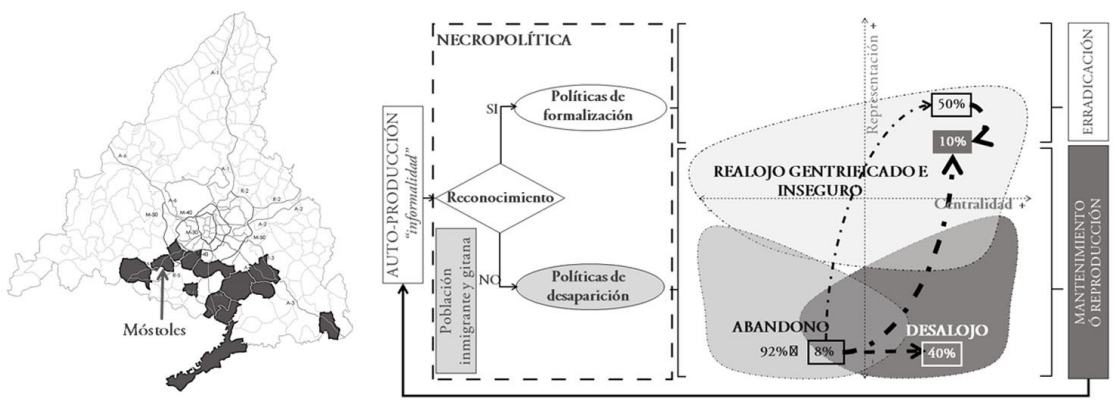
FIGURA 5 | Plano de situación de las viviendas de realojo y flujograma de las políticas implementadas
FUENTE: ELABORACIÓN PROPIA

Finalmente se llevó a cabo un proceso de realojo gentrificado e inseguro (figura 5), que implica para el $96 \%$ de los afectados mantener o reproducir su situación de informalidad. 


\section{De la coproducción a la necropolítica institucionalizada}

Según señalado en la introducción, se estima que, para el año 2020, vivirán en asentamientos informales 1392 millones de personas, el doble de lo que se esperaba en 1990 (un-Habitat, 2007, tabla B3, p. 352). En un contexto global de mercantilización y financiarización de la tierra y la vivienda, el acceso a estos bienes se hace inaccesible para una cada vez más amplia mayoría (Harvey, 2012; Rolnik, 2018). Esto ha contribuido al desarrollo de formas "alternativas" de autoproducción del espacio ("informales") (Alfaro et al., 2018; Davis, 2001; Vaccotti, 2017), proceso convertido para muchas personas en el único modo de acceso a dichos bienes, aunque sea en condiciones muy precarias (Secchi, 2013).

En este contexto, se han analizado las políticas llevadas a cabo en la CM, en España, en relación con los asentamientos autoproducidos desde 1979 a 2019. A partir de la teoría de la estructuración de Giddens (1984), se han analizado diversos procesos (tabla 1), lo que ha permitido extraer las siguientes conclusiones en relación con las preguntas planteadas en el método:

- Se ha mostrado cómo, en todos los casos, las políticas y acciones han ido encaminadas a erradicar las formas autogestionadas (informales) de producción del espacio (Procacci, 1991; Valverde Gefaell, 2015).

- Además, se ha mostrado cómo los planes implementados en relación con estos asentamientos han venido determinados por el valor que tenían las comunidades afectadas para el poder establecido (necropolítica):

- Cuando la comunidad tenía valor para el poder establecido, como fue el caso de la clase obrera en el contexto de una democracia recién estrenada, se ha implementado una política de "formalización" (figura 3); en el caso analizado, mediante un Plan coproducido de Remodelación de Barrios, que permitió el realojo in situ de la comunidad en condiciones adaptadas y asequibles (tabla 1).

- Sin embargo, cuando se ha considerado que la comunidad no tenía valor para el poder establecido, al no tener cabida en las formas de producción y consumo del sistema capitalista, como ha sido el caso de la comunidad gitana en Madrid, se han implementado políticas para "forzar su desaparición"; en los casos analizados (CRPM, IRIs y AVs en Las Sabinas), mediante procesos cíclicos de abandono institucional y planes de realojo gentrificados e inseguros (figuras 4 y 5), que han implicado el mantenimiento la reproducción de la "informalidad" que supuestamente se quería erradicar. 


\begin{tabular}{|c|c|c|c|c|}
\hline & PRB & CRPM & IRIS & AVS - LAS SABINAS \\
\hline & (I979-I998) & (I986-I998) & (1998-2015) & $(2013-2019)$ \\
\hline Objetivo & $\begin{array}{l}\text { "¡Vivienda digna, } \\
\text { aquí y ahora!" }\end{array}$ & $\begin{array}{l}\text { "Erradicación del } \\
\text { chabolismo" }\end{array}$ & $\begin{array}{l}\text { "Realojamiento e } \\
\text { Integración social" }\end{array}$ & “Desmantelamiento" \\
\hline $\begin{array}{l}\text { Actores } \\
\text { involucrados } \\
\text { en la toma de } \\
\text { decisiones }\end{array}$ & $\begin{array}{l}\text { Los afectados } \\
\text { lideran el proceso a } \\
\text { través de las asocia- } \\
\text { ciones de vecinos. } \\
\text { Se coordinan con la } \\
\text { administración local } \\
\text { (Ayuntamiento) y } \\
\text { estatal (Ministerio } \\
\text { de Vivienda). }\end{array}$ & $\begin{array}{l}\text { Administración lo- } \\
\text { cal (Ayuntamiento). } \\
\text { Sin participación } \\
\text { alguna por parte de } \\
\text { los afectados. }\end{array}$ & $\begin{array}{l}\text { Administración re- } \\
\text { gional (Comunidad } \\
\text { de Madrid) y local } \\
\text { (Ayuntamiento). Se } \\
\text { limita la partici- } \\
\text { pación de quienes } \\
\text { cumplen los requisi- } \\
\text { tos a la selección de } \\
\text { entre tres posibles } \\
\text { viviendas. }\end{array}$ & $\begin{array}{l}\text { Administración re- } \\
\text { gional (Comunidad } \\
\text { de Madrid) y local } \\
\text { (Ayuntamiento). Sin } \\
\text { participación alguna } \\
\text { por parte de los } \\
\text { afectados. }\end{array}$ \\
\hline \multirow{3}{*}{$\begin{array}{l}\text { Acceso a la } \\
\text { ciudad }\end{array}$} & $\begin{array}{l}\text { Realojo colectivo e } \\
\text { IN-SITU }\end{array}$ & \begin{tabular}{|l|} 
Realojo en guetos \\
segregados $55 \%$ en \\
BTE y CP
\end{tabular} & $\begin{array}{l}\text { Realojo disperso } \\
\mathbf{9 0 \%} \text { en la periferia } \\
\text { del sur-este }\end{array}$ & $\begin{array}{l}\text { Realojo disperso } \\
84 \% \text { en la periferia } \\
\text { del sur-este } \\
\end{array}$ \\
\hline & $\begin{array}{l}\text { Integrado en la } \\
\text { ciudad. } 5 \mathrm{~km} \text { de } \\
\text { media del centro de } \\
\text { Madrid. } \\
\end{array}$ & $\begin{array}{l}\text { Ni se plantea } \\
\text { la necesidad de } \\
\text { integración en la } \\
\text { ciudad. }\end{array}$ & $\begin{array}{l}\text { Integrado en } \\
\text { núcleos urbanos. } \\
15 \mathrm{~km} \text { de media del } \\
\text { centro de Madrid. } \\
\end{array}$ & $\begin{array}{l}\text { Integrado en núcleos } \\
\text { urbanos. } 50 \mathrm{~km} \text { de } \\
\text { media del centro de } \\
\text { Madrid. }\end{array}$ \\
\hline & Bien equipado & Sin equipamientos & Bien equipado & $\begin{array}{l}\text { Dependiendo del } \\
\text { núcleo urbano }\end{array}$ \\
\hline \multirow{4}{*}{$\begin{array}{l}\text { Acceso a la } \\
\text { vivienda }\end{array}$} & $\begin{array}{l}\text { Solución } \\
\text { adaptada } \\
\text { caso a caso }\end{array}$ & $\begin{array}{l}\text { Solución } \\
\text { No adaptada }\end{array}$ & $\begin{array}{l}\text { Elección entre tres } \\
\text { viviendas atendien- } \\
\text { do a sus lugares de } \\
\text { preferencia }\end{array}$ & $\begin{array}{l}\text { Solución } \\
\text { No adaptada }\end{array}$ \\
\hline & Viviendas de calidad & $\begin{array}{l}45 \% \text { viviendas } \\
\text { provisionales }\end{array}$ & Viviendas de calidad & Viviendas de calidad \\
\hline & $\begin{array}{l}\text { Asequible } \\
(10 \% \text { ingresos })\end{array}$ & - & $\begin{array}{l}\text { No-asequible } \\
(+50 \% \text { ingresos })\end{array}$ & $\begin{array}{l}\text { NO-asequible } \\
(+50 \% \text { ingresos })\end{array}$ \\
\hline & $\begin{array}{l}\text { Segura } \\
\text { (en propiedad) }\end{array}$ & $\begin{array}{l}\text { IN-segura } \\
\text { (45\% fueron des- } \\
\text { mantelados) }\end{array}$ & $\begin{array}{l}\text { IN-segura } \\
\text { (en alquiler) }\end{array}$ & $\begin{array}{l}\text { IN-segura } \\
\text { (en alquiler) }\end{array}$ \\
\hline $\begin{array}{l}\text { Tipo de } \\
\text { vínculo }\end{array}$ & Coproducción & $\begin{array}{l}\text { Ruptura institu- } \\
\text { cional }\end{array}$ & $\begin{array}{l}\text { Ruptura institu- } \\
\text { cional }\end{array}$ & Ruptura institucional \\
\hline \multicolumn{5}{|c|}{$\begin{array}{l}\text { Afectados, excluidos y recursos destinados (estimaciones realizadas a partir de la información disponible } \\
\text { para esta investigación) }\end{array}$} \\
\hline $\begin{array}{l}\text { Afectados } \\
\text { (Hog.) }\end{array}$ & 40.000 & 4.690 & 6.040 & 375 \\
\hline $\begin{array}{l}\text { Excluidos } \\
\text { (Hog.) }\end{array}$ & 1.000 & 3.059 & 4.040 & 345 \\
\hline Excluida (\%) & $3 \%$ & $65 \%$ & $67 \%$ & $92 \%$ \\
\hline $\begin{array}{l}\text { Pisos otor- } \\
\text { gados }\end{array}$ & 39.000 & 902 & 2.000 & 40 \\
\hline Pisos/año & 2.053 & 75 & 250 & 8 \\
\hline BTE O CP & 0 & 719 & 0 & 0 \\
\hline$(€ / \mathrm{Viv})$ & 50.000 & 49.352 & 110.000 & 114.400 \\
\hline
\end{tabular}

TABLA I | Resultados comparados de los casos de estudio

FUENTE: ELABORACIÓN PROPIA 
Si bien los resultados obtenidos en esta investigación son contexto-dependientes, se considera que contribuyen a mostrar un proceso "biopolítico" (Foucault, 2003); es decir, un modo de ejercer poder sobre la vida de las personas mediante unas prácticas implementadas desde las administraciones públicas que, lejos de acabar con la pobreza y muchos menos con las causas estructurales que la generan, imposibilitan y fuerzan la desaparición de las formas autogestionadas de estas comunidades "no rentables" que, sin quererlo y sin saberlo siquiera, solo existiendo, evidencian la crueldad del neoliberalismo y sus desigualdades (Mbembe, 2003; Valverde Gefaell, 2015), y subvierten el proyecto social, político y económico dominante (Procacci, 1991).

Pese a que, en el Norte Global, estos espacios autoproducidos (informales) son meramente residuales, no dejan por ello de suponer un desafío a los intereses del capital y una transgresión a las normas impuestas por el Estado (García-Calderón \& Janoschka, 2016) e interpelan a uno de los pilares fundamentales del sistema de acumulación capitalista: la propiedad privada (Lopes de Souza, 2012).

Finalmente, en este trabajo se aboga por superar la dualidad implícita en el término "informalidad", y transitar hacía una redefinición del fenómeno, entendiéndolo como un proceso de negociación entre actores (Herrle \& Fokdal, 2011); un proceso marcado por la desigualdad, con lo que ello implica de constreńimiento y reducción de la capacidad de influir en los procesos de toma de decisiones (Alfaro et al., 2018). Como se viene señalando históricamente, la ciudad sigue siendo el reflejo de una lucha entre fuerzas de poder desiguales (Harvey, 2012; Jenkins et al., 2007; Lefebvre 1969); una lucha continua entre prácticas institucionalizadas y de autoorganización (García-Calderón \& Janoschka, 2016), cuyo desenlace viene determinado por el lugar que ocupa una comunidad dentro del espacio social y el modo de vinculación que logra generar (Álvarez et al., 2019). Esta cuestión, menos madura en Espańa, se ha venido debatiendo ampliamente en los estudios sobre marginalidad en Latinoamérica, desde la segunda mitad del siglo xx hasta nuestros días (Abufhele, 2019; Fernandes, 2008; Oliver, 1980; Quijano, 1972, entre otros muchos), entendiéndose que la superación de la marginalidad implica la transformación del conjunto de la sociedad, una transformación en la que los marginalizados tiene un papel protagónico (Cortés, 2017, p. 26).

\section{Referencias bibliográficas}

Abufhele, V. (2019). La política de la pobreza y el gobierno de los asentamientos informales en Chile. EURE, 45(135), 49-69. http://dx.doi.org/10.4067/S025071612019000200049

Albrechts, L. (2013). Reframing strategic spatial planning by using a coproduction perspective. Planning Theory, 12(1), 46-63. https://doi.org/10.1177/1473095212452722

Alfaro d'Alencon, P., Smith, H., Álvarez de Andrés, E., Cabrera, C., Lombard, M., Mazzolini, A., Michelutti, E., Moretto, L., \& Amandine, S. (2018). Interrogating informality: Conceptualisations, practices and policies in the light of the New Urban Agenda. Habitat International, 75, 59-66. https://doi.org/10.1016/j.habitatint.2018.04.007 
Álvarez de Andrés, E., Fernández Güell, J. M., \& Smith, H. (2015). Instruments for analysing urban conflicts in the Global South: The case of urban transformation processes in Dakar. Habitat International, 49, 187-196. https://doi.org/10.1016/j. habitatint.2015.05.021

Álvarez de Andrés, E., Cabrera, C., \& Smith, H. (2019). Resistance as resilience: A comparative analysis of state-community conflicts around self-built housing in Spain, Senegal and Argentina. Habitat International, 86, 116-125. https://doi.org/10.1016/j. habitatint.2019.03.003

Batley, R. \& Mcloughlin, C. (2010). Engagement with non-state service providers in fragile States: Reconciling state-building and service delivery. Development Policy Review, 28(2), 131-154. https://doi.org/10.1111/j.1467-7679.2010.00478.x

Bey, H., (1991). Zona Temporalmente Autónoma. Madrid: Enclave Libros.

Carmona, P. \& Rodríguez, E. (2007). Barrios: planificación, inmigración y movimiento vecinal (1939-1986). En Observatorio Metropolitano (eds.), Madrid ¿la suma de todos?: globalización, territorio, desigualdad (pp. 333-390). Madrid: Traficantes de Sueños.

Castro, P. \& Molina, J. (1996). Un ejemplo de participación y renovación urbana: la remodelación de barrios en Madrid (España). Experiencia seleccionada en el Concurso de Buenas Prácticas patrocinado por Dubái en 1996: Ciudades para un Futuro más Sostenible. http://habitat.aq.upm.es/dubai/96/bp258.html

Cortés, A. (2017). Aníbal Quijano: Marginalidad y urbanización dependiente en América Latina. Polis. Revista Latinoamericana, (46). https://journals.openedition.org/ polis/12348

Davis, M. (2001). Magical urbanism: Latinos reinvent the Us City. New York: Verso.

De Soto, H. (2000). El misterio del capital. Por qué el capitalismo triunfa en Occidente y fracasa en el resto del mundo. Lima: Empresa Editora El Comercio.

Devas, N., Amis, P., Beall, J., Grant, U., Mitlin, D., Rakodi, C., \& Satterthwaite, D. (2001). Urban governance and poverty: lessons from a study of ten cities in the South. University of Birmingham, International Development Department.

Durand-Lasserve, A. \& Royston, L. (2002): Holding their ground: secure land tenure for the urban poor in developing countries. London: Earthscan.

El Diario (2018, dic. 29). La venta de 1.860 viviendas sociales a fondos buitre que condenó al Gobierno de Ana Botella. https://www.eldiario.es/madrid/Cronologia-viviendascelebracion-perjuicio-municipales_0_851465094.html

El Mundo (2015, mar. 7). Envejecer esperando un realojo. http://www.elmundo.es/ madrid/2015/03/07/54fb73e3e2704ee8558b4578.html

El Plural (2913, jul. 20). La Comunidad ha reducido programas contra la exclusión y de realojamiento y ha cerrado centros de inserción laboral. https://www.elplural. $\mathrm{com} /$ politica/la-comunidad-ha-reducido-programas-contra-la-exclusion-y-derealojamiento-y-ha-cerrado-centros-de-insercion-laboral_61355102

Fernandes, E. (2008). Consideraciones generales sobre las políticas públicas de regularización de asentamientos informales en América Latina. EURE, 34(102), 25-38. http://dx.doi. org/10.4067/S0250-71612008000200002

Foucault, M. (2003). Society must be defended: lectures at the College de France 1975-1976. New York: Picador. 
Frediani, A. A. (2007). Housing freedom, Amartya Sen and urban development policies-squatter settlement upgrading in Salvador da Bahia, Brazil. Ph.D. Thesis, Oxford Brookes University, uK.

García-Calderón Pavón, I. \& Janoschka, M. (2016). Viviendas en disputa ¿Espacios de emancipación? Un análisis de las luchas por la vivienda en Madrid. Historia Actual Online, 40(2), 113-127.

Giddens, A. (1984). The constitution of society: outline of the theory of structuration. Cambridge, uk: Polity Press.

Gutiérrez, A. \& Vives-Miró, S. (2018). Acumulación de viviendas por parte de los bancos a través de los desahucios: Geografía de la desposesión de vivienda en Cataluña. EURE, 44(132), 5-26. http://dx.doi.org/10.4067/s0250-71612018000200005

Harvey, D. (2012). Rebel cities: From the right to the city to the urban revolution. London: Verso.

Herrle, P. \& Fokdal, J. (2011). Beyond the urban informality discourse: Negotiating power, legitimacy and resources. Geographische Zeitschrift, 99(1), 3-15. https://www.jstor.org/ stable/23226577

Jenkins, P., Smith, H., \& Wang, Y. P. (2007). Planning and housing in the rapidly urbanising world. New York: Routledge.

Lago Ávila, M. (2014). El otro Madrid: el chabolismo que no cesa. Actuación autonómica en políticas de realojamiento e integración social 1997-2010. Estudios Geográficos, 75(276), 219-260. http://dx.doi.org/10.3989/estgeogr.201406

Lefebvre, H. (1969). El derecho a la ciudad. Barcelona: Península.

Lopes de Souza, M. (2012). Challenging heteronomous power in a globalized world. En S. Krätke, K. Wildner, \& S. Lanz (eds.), Transnationalism and urbanism (pp. 172-196). New York: Routledge.

Martínez, M. \& García, A. (2015). Ocupar las plazas, liberar edificios. Acme, 14(1).

Max-Neef, M., Elizalde, A., \& Hopenhayn, M. (2010). Desarrollo a escala humana: Una opción para el futuro. Madrid: Biblioteca $\mathrm{CF}+\mathrm{S}$.

Mbembe, A. (2003). Necropolitics. Public Culture, 15(1), 11-40. https://doi. org/10.1215/08992363-15-1-11

Mitlin, D. (2008). With and beyond the state - co-production as a route to political influence, power and transformation for grassroots organizations. Environment and Urbanization, 20(2), 339-360. https://doi.org/10.1177/0956247808096117

Mitlin, D. \& Bartlett, S. (2018). Editorial: Co-production - key ideas. Environment and Urbanization, 30(2), 355-366. https://doi.org/10.1177/0956247818791931

Montes, J. (1994). Sobre el realojamiento de los gitanos. En T. San Román (ed.), Entre la marginación y el racismo. Reflexiones sobre la vida de los gitanos (pp. 155-170). Madrid: Alianza.

Netto, G., Fitzpatrick, S., Sosenko, F., \& Smith, H. (2015). International lessons on tackling extreme housing exclusion. York, uk: Joseph Rowntree Foundation.

Nickson, A. \& Franceys, R. (2003). Tapping the market. The challenge of institutional reform in the urban sector. Houndmills \& New York: Palgrave.

Nogués Sáez, L. (2010). Exclusión residencial y políticas públicas: el caso de la minoría gitana en Madrid (1986-2006). Granada: Editorial de la Universidad de Granada.

Oliven, R. G. (1980). Marginalidad urbana en América Latina. EURE, 7(19), 49-62. http:// www.eure.cl/index.php/eure/article/download/896/11 
Piketty, T. (2015). La economía de las desigualdades. Buenos Aires: Siglo XxI.

Portes, A., Roberts, B. R., \& Grimson, A. (2005). Ciudades latinoamericanas: un análisis comparativo en el umbral del nuevo siglo. Buenos Aires: Prometeo.

Procacci, G. (1991). Social economy and the government of poverty. En M. Foucault, G. Burchell, C. Gordon, \& P. Miller (eds.), The Foucault effect: studies in governmentality: with two lectures by and an interview with Michel Foucault (Ch. 7). Chicago, IL: University of Chicago Press.

Quijano, A. (1972). La constitución del "mundo" de la marginalidad urbana. EURE, 5, 89106. http://www.eure.cl/index.php/eure/article/viewFile/837/685

Rodríguez-Villasante, T., Alguacil, K., Denche, C., Hernández Aja, A., León, C., \& Velázquez, I. (2009). Retrato de chabolista con piso. Análisis de redes sociales en la remodelación de barrios de Madrid. Madrid: ALFOZ-CIDUR/ IVIMA-SGV.

Rolnik, R. (2018). La guerra de los lugares: La colonización de la tierra y la vivienda en la era de las finanzas. Barcelona: Descontrol.

Roy, A. (2010). Informality and the politics of planning. En J. Hillier \& P. Healey (eds.), The Ashgate research companion to planning theory: Conceptual challenges for spatial planning (pp. 87-107). Farnham, uk: Ashgate Publishing Co.

Roy, A. (2011). Slumdog cities: Rethinking subaltern urbanism. International Journal of Urban and Regional Research, 35(2), 223-238. https://doi.org/10.1111/j.14682427.2011.01051.x

Roy, A. \& AlSayyad, N. (eds.). (2004). Urban informality. Transnational perspectives from the Middle East, Latina America, and South Asia. Lanham, MD: Lexington Books.

Satterthwaite, D. \& Mitlin, D. (2014). Reducing urban poverty in the global South. London \& New York: Routledge.

Secchi, B. (2013). La città dei ricchi e la città dei poveri. Roma / Bari: Laterza.

Shiva, V. (2005). Hacer que la pobreza sea historia, y la Historia de la Pobreza. Como poner fin a la pobreza [En línea]. Rebelión. http://www.rebelion.org/noticia.php?id=15959

Turner, J. F. C. (1967). Barriers and channels for housing development in modernizing countries. Journal of the American Institute of Planners, 33(3), 167-180. https://doi. org/10.1080/01944366708977912

Turner, J. F. C. (1968). Housing priorities, settlements patterns and urban development in modernizing countries. Journal of the American Institute of Planners, 34(6), 354-363.

Turner, J. F. C. (1972). Housing as a verb. En J. F. C. Turner \& R. Fichter (eds.), Freedom to build. Dweller control of the housing process (pp. 148-175). New York: Collier Macmillan. http://www.communityplanning.net/JohnTurnerArchive/pdfs/FreedomtoBuildCh7. pdf

un-Habitat (2007). Global Report on Human Settlements 2007. Enhancing Urban Safety and Security. London \& Sterling, va: un-Habitat / Earthscan. https://www.un.org/ ruleoflaw/files/urbansafetyandsecurity.pdf

Vaccotti, L. (2017). Migraciones e informalidad urbana. Dinámicas contemporáneas de la exclusión y la inclusión en Buenos Aires. EURE, 43(129), 49-70. http://dx.doi. org/10.4067/S0250-71612017000200003

Valverde Gefaell, C. (2015). De la necropolítica neoliberal a la empatía radical: violencia discreta, cuerpos excluidos y repolitización. Barcelona: Icària. 
Varley, A. (2013). Postcolonialising informality? Environment and Planning D, 31(1), 4-22. https://doi.org/10.1068/d14410

Verschuere, B., Brandsen, T., \& Pestoff, V. (2012). Co-production: The state of the art in research and the future agenda. Voluntas, 23(4), 1083-1101. https://doi.org/10.1007/ s11266-012-9307-8

Watson, V. (2009). Seeing from the South: Refocusing urban planning on the globe's central urban issues. Urban Studies, 46(11), 2259-2275. https://doi. org/10.1177/0042098009342598

Wild, L., Chambers, V., King, M., \& Harris, D. (2012). Common constraints and incentive problems in service delivery. Overseas Development Institute (ODI) Working Paper 351. London: oDI. https://www.odi.org/sites/odi.org.uk/files/odi-assets/publicationsopinion-files/7791.pdf

Yin, R. K. (1994). Case study research: Design and methods. Thousand Oaks, Ca: Sage. 